Pacific

Journal of

Mathematics

\title{
DERIVED CATEGORIES OF REPRESENTATIONS OF SMALL CATEGORIES OVER COMMUTATIVE NOETHERIAN RINGS
}

Benjamin Antieau And Greg Stevenson

Volume $283 \quad$ No. 1

July 2016 


\title{
DERIVED CATEGORIES OF REPRESENTATIONS OF SMALL CATEGORIES OVER COMMUTATIVE NOETHERIAN RINGS
}

\author{
Benjamin Antieau And Greg Stevenson
}

\begin{abstract}
We study the derived categories of small categories over commutative noetherian rings. Our main result is a parametrization of the localizing subcategories in terms of the spectrum of the ring and the localizing subcategories over residue fields. In the special case of representations of Dynkin quivers over a commutative noetherian ring, we give a complete description of the localizing subcategories of the derived category and a complete description of the thick subcategories of the perfect complexes. We also show that the telescope conjecture holds in this setting and we present some results concerning the telescope conjecture more generally.
\end{abstract}

\section{Introduction}

If $\mathrm{T}$ is a triangulated category with all coproducts, a localizing subcategory $\mathrm{L} \subseteq \mathrm{T}$ is a full triangulated subcategory closed under all coproducts in T. Localizing subcategories are so-named because in good cases (the Bousfield localizations) the Verdier quotient functor $\mathrm{T} \rightarrow \mathrm{T} / \mathrm{L}$ possesses a right adjoint, i.e., they give rise to localization functors. Understanding the collection of localizing subcategories on a given triangulated category is a challenging and interesting problem which has been completely resolved in only a few classes of examples.

The history of such problems has roots in stable homotopy theory, where one would like to relate two localizations of the $p$-local stable homotopy category $\mathrm{SH}_{(p)}$ : one which has excellent theoretical properties (localization with respect to the homology theory given by the Johnson-Wilson spectrum $\mathrm{E}(n))$ and one which is computable (the telescopic localization). The importance of such questions arose first in [Bousfield 1979] and [Ravenel 1984]. That these two localizations agree is the still-open telescope conjecture. Work on nilpotence closely related to the telescope

Antieau was supported by NSF grant number DMS-1461847. Stevenson was partly supported by NSF grant number 0932078000 while in residence at the Mathematical Science Research Institute and by the Alexander von Humboldt Stiftung.

MSC2010: primary 16E35, 16G20; secondary 13D09, $18 \mathrm{G} 55$.

Keywords: derived categories, localizations, telescope conjecture. 
conjecture by Devinatz, Hopkins, and Smith [Devinatz et al. 1988; Hopkins and Smith 1998] has led to the classification of all thick subcategories, i.e., triangulated subcategories closed under direct summands, of $\mathrm{SH}^{\text {fin }}$, the homotopy category of finite spectra. Using similar ideas on the detection of nilpotent maps between objects in $\mathrm{D}(R)$, Neeman [1992] classified the localizing subcategories of $\mathrm{D}(R)$ and the thick subcategories of $\mathrm{D}^{\text {perf }}(R)$ when $R$ is noetherian in terms of Spec $R$.

Going beyond the example of $\mathrm{D}(R)$ where $R$ is noetherian and commutative seems rather difficult. In terms of classification of thick subcategories of $\mathrm{D}^{\text {perf }}(X)$, when $X$ is a quasicompact and quasiseparated scheme, one has the result of [Thomason 1997], which says that the thick subcategories which are also tensor ideals correspond bijectively to unions of closed subsets of $X$ with quasicompact complement. This kind of result has been taken up by other authors, such as Benson, Carlson, and Rickard [Benson et al. 1997] and Benson, Iyengar, and Krause [Benson et al. 2011], who study the tensor ideals of stable module categories of finite groups. This is part of a generalized framework of studying tensor ideals, pursued by Balmer [2005], Dell'Ambrogio and Stevenson [2013; 2014], and Stevenson [2013; 2014].

In contrast to all that is known about thick subcategories, very little is known about localizing subcategories outside of Neeman's theorem. For instance, one does not know all localizing subcategories of $\mathrm{D}_{\mathrm{qc}}\left(\mathbb{P}_{\mathbb{C}}^{1}\right)$. We mention one more example, due to Brüning [2007], who classified the localizing subcategories of $\mathrm{D}(A)$ where $A$ is a hereditary Artin algebra of finite representation type.

Let $R$ be a noetherian commutative ring. We show that in many cases classification of the localizing subcategories of an $R$-linear triangulated category can be reduced to studying the localizing subcategories of the "fibers" over the residue fields of $R$.

Let $C$ be a small category, and let $s: \mathcal{L} \rightarrow$ Spec $R$ denote the class constructed fiber by fiber over Spec $R$, by letting $s^{-1}(p)$, for $p \in \operatorname{Spec} R$, be the class of localizing subcategories of $\mathrm{D}(k(p) C)$. Note that, a priori, the localizing subcategories of $\mathrm{D}(k(p) C)$ only form a proper class, which is the reason for the careful wording above. There is, however, no known example of a compactly generated triangulated category whose collection of localizing subcategories does not form a set. The following result is our first theorem.

Theorem (Corollary 4.3). Let $R$ be a noetherian commutative ring and $C$ a small category. Then there is an isomorphism of lattices

$\{$ localizing subcategories $\mathrm{L}$ of $\mathrm{D}(R C)\} \underset{g}{\stackrel{f}{\rightleftarrows}}\{$ sections l of $\mathcal{L} \stackrel{s}{\longrightarrow} \operatorname{Spec} R\}$,

where $f$ takes a localizing subcategory $\mathrm{L}$ of $\mathrm{D}(R C)$ to the function $l: \operatorname{Spec} R \rightarrow \mathcal{L}$ such that $l(p)=\operatorname{add}\left(k(p) \otimes_{R} \mathrm{~L}\right)$, and where $g(l)$ is the localizing subcategory generated by all $X$ such that $k(p) \otimes_{R} X \in l(p)$ for all $p \in \operatorname{Spec} R$. 
In fact, our methods apply somewhat more generally, allowing one to replace $\mathrm{D}(R C)$ with derived categories of representations of $R$-flat $R$-linear categories.

Our second result is a classification of the telescopic localizations of $\mathrm{D}(R Q)$ and a classification of the thick subcategories of $\mathrm{D}^{\text {perf }}(R Q)$ when $Q$ is a Dynkin quiver.

Theorem (Corollaries 5.1, 5.10, and 5.11). Let $R$ be a noetherian commutative ring and $Q$ a simply laced Dynkin quiver, and denote by $R Q$ the $R$-linear path algebra of $Q$. There is an isomorphism of lattices

$\{$ localizing subcategories of $\mathrm{D}(R Q)\} \underset{g}{\stackrel{f}{\rightleftarrows}}\{$ functions $\operatorname{Spec} R \rightarrow \mathrm{NC}(Q)\}$,

where $\mathrm{NC}(Q)$ denotes the lattice of noncrossing partitions associated to $Q$.

Moreover, the telescope conjecture holds for $\mathrm{D}(R Q)$, and the smashing subcategories, which by virtue of the telescope conjecture are in bijection with thick subcategories of $\mathrm{D}^{\text {perf }}(R Q)$, correspond to those $\sigma: \operatorname{Spec} R \rightarrow \mathrm{NC}(Q)$ such that whenever $p \subseteq q$ in Spec $R$ we have $\sigma(p) \leq \sigma(q)$.

In terms of the localizing subcategories, this theorem basically combines Corollary 4.3 with the results of [Ingalls and Thomas 2009] on localizing subcategories of $\mathrm{D}(k Q)$ for fields $k$.

Initially, we had also hoped to prove the telescope conjecture for the telescopic localizations of $\mathrm{D}(R C)$ more generally, at least with some hopefully mild hypothesis. This turned out to be overly ambitious, but we present some partial results in Section 6.

\section{Preliminaries on representations of small categories}

Throughout we fix a commutative ring $R$. Let $C$ be a small category.

Definition 2.1. The category of right $C$-modules over $R$ is the functor category

$$
\operatorname{Mod}_{R} C=\operatorname{Fun}\left(C^{\text {op }}, \operatorname{Mod} R\right)
$$

consisting of contravariant functors from $C$ to the category of $R$-modules.

The following well-known lemma ensures that we can use the standard tools of homological algebra when dealing with $C$-modules.

Lemma 2.2. The category $\operatorname{Mod}_{R} C$ of right $C$-modules over $R$ is a Grothendieck category with enough projectives.

Proof. Recall that a Grothendieck (abelian) category is an abelian category (1) satisfying axiom (AB5), on the existence and exactness of filtered colimits, and (2) possessing a generator. The lemma can be proved by showing that the direct sum of the set of representable objects is a generator, that filtered colimits are 
computed pointwise so that (AB5) follows from the satisfaction of that axiom for $\operatorname{Mod}_{R}$ itself, and finally that the projective objects of $\operatorname{Mod}_{R} C$ are summands of direct sums of representables. Details are left to the reader.

We can also approach $C$-modules via $R$-linear functors.

Definition 2.3. The $R$-linearization of $C$, which we will denote by $R C$, is the category with the same objects as $C$ and whose hom-objects are free $R$-modules on the hom-sets of $C$

$$
R C\left(c, c^{\prime}\right)=\bigoplus_{f \in C\left(c, c^{\prime}\right)} R f
$$

with the obvious composition rule. In other words, $R C$ is the free $R$-linear category on $C$.

Definition 2.4. An $R$-linear category $D$ is a small category enriched in $R$-modules. It is flat if $D\left(c, c^{\prime}\right)$ is a flat $R$-module for all pairs of objects $c, c^{\prime}$ in $D$.

Definition 2.5. If $D$ is an $R$-linear category, then the category of right $D$-modules over $R$ is defined to be the functor category

$$
\operatorname{Mod}_{R} D=\operatorname{Fun}_{R}(D, \operatorname{Mod} R)
$$

of $R$-linear functors.

Evidently, $R C$ is a flat $R$-linear category for any small category $C$, since the hom-objects are free. The reason for looking at these more general categories is to capture the representation theory of $R$-algebras "with many objects", whereas the representations of $R C$ are representations of monoids with many objects. In the case where $C$ has one object with monoid of endomorphisms $M$, the category of representations of $C$ in $R$-modules is equivalent to the category of right $R[M]$-modules, where $R[M]$ is the monoid algebra of $M$. On the other hand, if $D$ is an $R$-linear category with one object having endomorphism algebra $S$, then $S$ is an $R$-algebra, and the category of $R$-linear representations of $D$ is equivalent to the category of right $S$-modules. Of course, not every $R$-algebra is a monoid algebra, so the $R$-linear categories capture more examples.

Of course, we should now check that $\operatorname{Mod}_{R} C$ and $\operatorname{Mod}_{R} R C$ are equivalent. We do this in a moment, but we first want to introduce extra structure that will be preserved. Tensoring an $R C$-module objectwise with an $R$-module defines a bifunctor

$$
\operatorname{Mod} R \times \operatorname{Mod}_{R} R C \stackrel{\otimes_{R}}{\longrightarrow} \operatorname{Mod}_{R} R C
$$

which is explicitly given by $\left(M \otimes_{R} F\right)(c)=M \otimes_{R} F(c)$ for an $R$-module $M$, an $R C$-module $F$, and $c \in C$. This gives an action of the category of $R$-modules on the category of $R C$-modules. We note that this action is nothing other than the existence of copowers for the $R$-linear category $\operatorname{Mod}_{R} R C$. There is, of course, a similar action on $\operatorname{Mod}_{R} D$ when $D$ is an $R$-linear category. 
Remark 2.6. Here and in the sequel we will work with categories of the form $R C$ since our main examples are of this form. However, our results are equally valid for flat $R$-linear categories; the only changes which need to be made are cosmetic.

Lemma 2.7. The natural map $\operatorname{Mod}_{R} R C \rightarrow \operatorname{Mod}_{R} C$ is an equivalence for any small category $C$. This equivalence is compatible with the actions described above. Proof. This follows from the standard 2-adjunction relating categories and $R$-linear categories; see for instance [Kelly 1982, Chapter 2.5].

Lemma 2.8. Given a morphism of commutative rings $R \stackrel{\phi}{\longrightarrow} S$, the natural base change functor

$$
\phi^{*}: \operatorname{Mod}_{R} R C \rightarrow \operatorname{Mod}_{S} S C
$$

has a right adjoint $\phi_{*}$.

Proof. The functor $\phi^{*}$ is given by applying $S \otimes_{R}-$ objectwise, and $\phi_{*}$ is induced by restriction of scalars. This is again induced by a standard 2-adjunction between $R$-linear and $S$-linear categories corresponding to $\phi$.

\section{Generalities on derived categories of small categories over a commutative ring}

Again $R$ is a fixed commutative ring which we now also assume is noetherian, and $C$ is a small category with $R$-linearization $R C$. The (unbounded) derived category $\mathrm{D}(R C)$ of $R C$ is the category of complexes of right $R C$-modules where quasi-isomorphisms have been inverted. We note that this is a compactly generated triangulated category and the compact objects are, up to quasi-isomorphism, precisely the bounded complexes of projective $R C$-modules.

Recall that a localizing subcategory of $\mathrm{D}(R C)$ is a full triangulated subcategory of $\mathrm{D}(R C)$ closed under coproducts (any such subcategory is automatically closed under direct summands). We want to consider to what extent the localizing subcategories of $\mathrm{D}(R C)$ are determined by the localizing subcategories of $\mathrm{D}(k(p) C)$ as $p$ ranges over the prime ideals of $R$. This is inspired by work of Neeman [1992] who showed that in the case where $C$ is the terminal category, i.e., $R C=R$, the localizing subcategories of $\mathrm{D}(R)$ are determined by those of the $\mathrm{D}(k(p))$. We restrict to noetherian rings as, even in the case $R C=R$, it is known that $\operatorname{Spec} R$ does not determine the localizing subcategories of $\mathrm{D}(R)$ in general.

Let us begin by observing that the action of $\operatorname{Mod} R$ on $\operatorname{Mod}_{R} C$ can be derived:

Lemma 3.1. The bifunctor $\operatorname{Mod} R \times \operatorname{Mod}_{R} C \rightarrow \operatorname{Mod}_{R} C$ is left-balanced, with respect to flat $R$-modules and objectwise $R$-flat $R C$-modules, i.e., it is exact when either the first variable is flat or the second variable is objectwise flat. It admits a leftderived functor, independent up to isomorphism of which variable it is derived in, which gives a left action $\mathrm{D}(R) \times \mathrm{D}(R C) \rightarrow \mathrm{D}(R C)$ in the sense of [Stevenson 2013]. 
Proof. Given $F \in \operatorname{Mod}_{R} C$ such that $F$ is objectwise $R$-flat, it is clear that $-\otimes_{R} F$ is exact. As $\operatorname{Mod}_{R} C$ has enough projectives, and the projective $R C$-modules are componentwise projective, we see that $\operatorname{Mod}_{R} C$ has enough objectwise $R$-flat modules. It is thus clear that the functor can be left-derived, using resolutions either in $\operatorname{Mod} R$ or $\operatorname{Mod}_{R} C$, and that it does not matter, up to quasi-isomorphism, on which side the resolution is taken (i.e., $-\otimes_{R}-$ is balanced as claimed). It is straightforward to check that this gives an associative and unital action of $\mathrm{D}(R)$ on $\mathrm{D}(R C)$.

Remark 3.2. Given $E \in \mathrm{D}(R)$ and $F \in \mathrm{D}(R C)$, we will simply denote $E \otimes_{R}^{\mathrm{L}} F$ by $E \otimes_{R} F$ or even $E \otimes F$; no confusion should result as we will almost exclusively work with derived functors (frequently with $R$ fixed or clear from the context).

This allows us to utilize the machinery of tensor actions to analyze localizing subcategories of $\mathrm{D}(R C)$. After giving a convenient lemma and some notation, we will recall the main result that we will need from this theory.

Lemma 3.3. Any localizing subcategory $\mathrm{L} \subseteq \mathrm{D}(R C)$ is closed under tensoring with complexes of $R$-modules. Explicitly, for any $M \in \mathrm{D}(R)$ and $X \in \mathrm{L}$, we have $M \otimes_{R} X \in \mathrm{L}$.

Proof. Evidently, if $X \in \mathrm{L}$, then $R \otimes_{R} X \simeq X \in \mathrm{L}$. Since $-\otimes_{R} X$ preserves coproducts, it follows that the subcategory of $\mathrm{D}(R)$ consisting of complexes of $R$-modules $M$ such that $M \otimes_{R} X \in \mathrm{L}$ is localizing and contains $R$. Since $R$ is a compact generator of $\mathrm{D}(R)$, the lemma follows.

Let $f$ be an element of $R$. We denote by $K_{\infty}(f)$ the stable Koszul complex $R \rightarrow R_{f}$ of $f$, where the map is the canonical one. Given a prime ideal $p$ of $R$, we set

$$
K_{\infty}(p)=K_{\infty}\left(f_{1}\right) \otimes_{R} \cdots \otimes_{R} K_{\infty}\left(f_{n}\right),
$$

where $f_{1}, \ldots, f_{n}$ is a choice of generators for $p$. The resulting complex is independent of the choice of generators up to quasi-isomorphism (independence is usually left as an exercise but a proof can be found, for instance, in [Greenlees 1993, Lemma 2.3]).

Given $p \in \operatorname{Spec} R$, we define the object $\Gamma_{p} R$ to be $K_{\infty}(p) \otimes_{R} R_{p}$. We recall from [Stevenson 2013] that $\Gamma_{p} R \otimes_{R} \Gamma_{p} R \simeq \Gamma_{p} R$ and for $p \neq q$ in Spec $R$ we have $\Gamma_{p} R \otimes_{R} \Gamma_{q} R=0$.

Remark 3.4. In more familiar language, the object $K_{\infty}(p)$ corresponds to taking local cohomology with support in $V(p)$ in the sense that the local cohomology functor is isomorphic to $K_{\infty}(p) \otimes(-)$. Thus $\Gamma_{p} R$ can be thought of as corresponding to " $p$-localized local cohomology on $V(p)$ ". In general it differs from the residue field $k(p)$, which is rarely tensor idempotent. In certain situations, for instance if $R=\mathbb{Z}$, one can express $\Gamma_{p} R$ as a desuspension of a flat resolution of $E(k(p))$, the injective envelope of the residue field at $p$; for instance, given a prime $p \in \mathbb{Z}$, one 
has $\Gamma_{(p)} \mathbb{Z} \cong \Sigma^{-1} E(\mathbb{Z} / p \mathbb{Z})$. However, in general the precise relationship between $\Gamma_{p} R, k(p)$, and $E(k(p))$ seems to be more subtle.

As a final point of notation, we will use $\langle S\rangle$ to denote the smallest localizing subcategory of a triangulated category generated by some collection of objects $S$.

Theorem 3.5 [Stevenson 2013, Theorem 6.9]. Given an object $X$ of $\mathrm{D}(R C)$, there is an equality of localizing subcategories

$$
\langle X\rangle=\left\langle\Gamma_{p} R \otimes_{R} X \mid p \in \operatorname{Spec} R\right\rangle .
$$

It follows that $\Gamma_{p} R \otimes_{R} X \simeq 0$ for all prime ideals $p$ if and only if $X \simeq 0$.

Corollary 3.6. If $X \in \mathrm{D}(R C)$ is nonzero, then there is some prime ideal $p$ of $R$ such that $k(p) \otimes_{R} X$ is not zero.

Proof. By the theorem there is a $p$ such that $\Gamma_{p} R \otimes_{R} X$ is nonzero. The result now follows as $\left\langle\Gamma_{p} R\right\rangle=\langle k(p)\rangle$ in $\mathrm{D}(R)$ by [Neeman 1992, Section 2], which implies $k(p) \otimes_{R} X \simeq 0$ if and only if $\Gamma_{p} R \otimes_{R} X \simeq 0$.

We now turn to analyzing the localizing subcategories of $\mathrm{D}(R C)$ in terms of the "fibers" $\mathrm{D}(k(p) C)$ for $p \in \operatorname{Spec} R$. Let $\mathcal{L}$ be the class defined in the following way. It comes equipped with a surjective map $\mathcal{L} \stackrel{s}{\longrightarrow} \operatorname{Spec} R$, and the fiber over $p \in \operatorname{Spec} R$ is the class of localizing subcategories of $\mathrm{D}(k(p) C)$. We will define a pair of maps

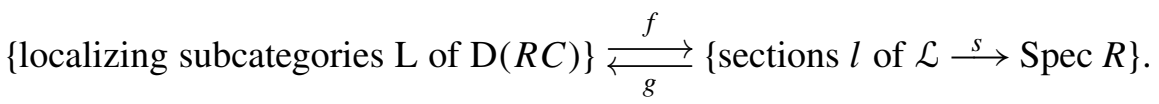

In order to define the maps in the most convenient manner, we require a little preparation.

Lemma 3.7. If $X$ is in the image of the forgetful functor $\mathrm{D}(k(p) C) \rightarrow \mathrm{D}(R C)$, then $k(p) \otimes_{R} X$ is a direct sum of suspensions of $X$. In particular, the base change functor $\mathrm{D}(R C) \rightarrow \mathrm{D}(k(p) C)$ is essentially surjective up to summands.

Proof. Let $X$ be as in the statement, i.e., $X$ is a complex of $k(p) C$-modules regarded as a complex of $R C$-modules. Then

$$
k(p) \otimes_{R} X \simeq\left(k(p) \otimes_{R} k(p)\right) \otimes_{k(p)} X
$$

is a coproduct of suspensions of $X$ since $k(p) \otimes_{R} k(p)$ is a coproduct of suspensions of $k(p)$. As the base change functor $\mathrm{D}(R C) \rightarrow \mathrm{D}(k(p) C)$ is just $k(p) \otimes_{R}-$, the final statement of the lemma is an immediate consequence.

Lemma 3.8. Let $\mathrm{L}$ be a localizing subcategory of $\mathrm{D}(R C)$. Then $\operatorname{add}\left(k(p) \otimes_{R} \mathrm{~L}\right)$, the closure of $k(p) \otimes_{R} \mathrm{~L}$ under summands and isomorphisms in $\mathrm{D}(k(p) C)$, is a localizing subcategory of $\mathrm{D}(k(p) C)$. 
Proof. It is evident that $\operatorname{add}\left(k(p) \otimes_{R} \mathrm{~L}\right)$ is closed under suspensions and coproducts in $\mathrm{D}(k(p) C)$ as derived base change is exact and coproduct-preserving. Thus it is sufficient to show that $\operatorname{add}\left(k(p) \otimes_{R} \mathrm{~L}\right)$ is closed under triangles. Suppose $X \rightarrow Y \rightarrow Z \rightarrow$ is a triangle with $X, Y \in \operatorname{add}\left(k(p) \otimes_{R} \mathrm{~L}\right)$. Without loss of generality we may assume $X, Y \in k(p) \otimes_{R} \mathrm{~L}$. By Lemma 3.3 the restrictions of $X$ and $Y$ lie in $\mathrm{L}$, so we deduce that the restriction of $Z$ lies in L. Hence $k(p) \otimes_{R} Z$ is in $k(p) \otimes_{R} \mathrm{~L}$ and using Lemma 3.7 we see that $Z$ is in $\operatorname{add}\left(k(p) \otimes_{R} \mathrm{~L}\right)$, proving the lemma.

The function $f$ is defined as follows: we set $f(\mathrm{~L})(p)=\operatorname{add}\left(k(p) \otimes_{R} \mathrm{~L}\right)$ which is localizing by Lemma 3.8. Given a section $l$ of $s$, define $g(l)$ as the localizing subcategory

$$
\left\{X \in \mathrm{D}(R C) \mid k(p) \otimes_{R} X \in l(p) \text { for all primes } p \in \operatorname{Spec} R\right\} .
$$

There is another natural function

$$
\text { \{localizing subcategories } \left.\mathrm{L} \text { of } \mathrm{D}(R C)\} \overleftarrow{g^{\prime}} \text { \{sections } l \text { of } \mathcal{L} \stackrel{s}{\longrightarrow} \operatorname{Spec} R\right\}
$$

defined as follows: let $g^{\prime}$ be the function that takes $l$ to the localizing subcategory generated by the objects $X$ of $l(p)$ for all $p$, viewed as $R C$-modules in the natural way, i.e.,

$$
g^{\prime}(l)=\langle l(p) \mid p \in \operatorname{Spec} R\rangle .
$$

Lemma 3.9. If $\mathrm{L}$ is a localizing subcategory of $\mathrm{D}(R C)$ then $g^{\prime}(f(\mathrm{~L})) \subseteq \mathrm{L} \subseteq g(f(\mathrm{~L}))$. Proof. The inclusion $\mathrm{L} \subseteq g(f(\mathrm{~L}))$ is clear:

$$
g(f(\mathrm{~L}))=\left\{X \in \mathrm{D}(R C) \mid k(p) \otimes_{R} X \in \operatorname{add}\left(k(p) \otimes_{R} \mathrm{~L}\right) \text { for all } p \in \operatorname{Spec} R\right\} \supseteq \mathrm{L} .
$$

To show the other inclusion, note that $g^{\prime}(f(\mathrm{~L}))$ is generated by $k(p) \otimes_{R} X$, as $X$ ranges over the objects of $\mathrm{L}$ and $p$ ranges over the primes of $R$. But, by Lemma 3.3, these are all in L.

Lemma 3.10. Suppose $l$ is a section of s. Then $f\left(g^{\prime}(l)\right)=l=f(g(l))$. In particular, $f$ is surjective.

Proof. The value of $f\left(g^{\prime}(l)\right)$ at a prime $p$ consists of the localizing subcategory of $\mathrm{D}(k(p) C)$ generated by the complexes $k(p) \otimes_{R} X$ for $X \in l(p)$. By Lemma 3.7 $k(p) \otimes_{R} X$ is a direct sum of suspensions of $X$ and thus $f\left(g^{\prime}(l)\right)=l$. Similarly $l=f(g(l))$, proving the lemma.

Our goal is to show that $g^{\prime}(f(\mathrm{~L}))=\mathrm{L}=g(f(\mathrm{~L}))$. This will prove that $g$ and $f$ are inverse bijections and so gives a description of the lattice of localizing subcategories of $\mathrm{D}(R C)$ in terms of the corresponding derived categories over the residue fields of $\operatorname{Spec} R$. 


\section{Proof of the main theorem}

This section is dedicated to proving $g^{\prime}(f(L))=L=g(f(L))$.

Write $\Gamma_{p} \mathrm{D}(R C)$ for the localizing subcategory consisting of objects $X$ supported at $p \in \operatorname{Spec} R$, i.e., those $X$ satisfying $k(q) \otimes_{R} X \simeq 0$ for $q \neq p$. Equivalently, one can describe $\Gamma_{p} \mathrm{D}(R C)$ as the essential image of $\Gamma_{p} R \otimes_{R}-$ in $\mathrm{D}(R C)$. We can restrict $f$ to the class of localizing subcategories of $\Gamma_{p} \mathrm{D}(R C)$.

Proposition 4.1. The following are equivalent:

(1) the functions $f$ and $g$ are inverse bijections;

(2) the restrictions

$$
\left\{\begin{array}{c}
\text { localizing subcategories } \\
\text { of } \Gamma_{p} \mathrm{D}(R C)
\end{array}\right\} \underset{g_{p}}{\stackrel{f_{p}}{\rightleftarrows}}\left\{\begin{array}{c}
\text { localizing subcategories } \\
\text { of } \mathrm{D}(k(p) C)
\end{array}\right\}
$$

are inverse bijections for all primes $p$;

(3) for every prime ideal $p$ in Spec $R$ and for every object $X$ of $\Gamma_{p} \mathrm{D}(R C)$, the localizing subcategories $\left\langle k(p) \otimes_{R} X\right\rangle$ and $\langle X\rangle$ are the same.

Proof. Clearly (1) implies (2). That (2) implies (3) follows from the fact that the localizing subcategories $\langle X\rangle$ and $\left\langle k(p) \otimes_{R} X\right\rangle$ have the same image under $f_{p}$. Since $f$ is surjective, to prove that (3) implies (1), it suffices to prove that (3) implies $f$ is injective. Assuming this for a moment, Lemma 3.10 says that both $g$ and $g^{\prime}$ are inverses for $f$, which must then coincide.

Assume now that $\mathrm{L}$ is a localizing subcategory of $\mathrm{D}(R C)$ and that $X \in \mathrm{L}$. It suffices to show that $X \in g^{\prime}(f(\mathrm{~L}))$ since we have the other containment by Lemma 3.9. Under the assumption (3), $\Gamma_{p} R \otimes_{R} X \in g^{\prime}(f(\mathrm{~L}))$ for every prime ideal $p$ in Spec $R$ because $k(p) \otimes_{R} \Gamma_{p} R \otimes_{R} X \cong k(p) \otimes_{R} X$. Hence there is a containment of localizing subcategories

$$
\left\langle\Gamma_{p} R \otimes_{R} X \mid p \in \operatorname{Spec} R\right\rangle \subseteq g^{\prime}(f(\mathrm{~L})) .
$$

By Theorem 3.5, $X \in\left\langle\Gamma_{p} R \otimes_{R} X\right| p \in$ Spec $\left.R\right\rangle$, and so $X \in g^{\prime}(f(\mathrm{~L}))$, completing the proof.

The following observation is our main 'theorem'.

Theorem 4.2. Let $p$ be a prime ideal of $R$ and $X$ an object of $\Gamma_{p} \mathrm{D}(R C)$. Then $X \in\left\langle k(p) \otimes_{R} X\right\rangle$ and hence

$$
\left\langle k(p) \otimes_{R} X\right\rangle=\langle X\rangle .
$$

Proof. Let $X$ be as in the lemma and consider the full subcategory

$$
\mathrm{M}=\left\{E \in \mathrm{D}(R) \mid E \otimes_{R} X \in\left\langle k(p) \otimes_{R} X\right\rangle\right\}
$$


of $\mathrm{D}(R)$. As $\left\langle k(p) \otimes_{R} X\right\rangle$ is a localizing subcategory, it follows that $\mathrm{M}$ is also localizing (this is relatively straightforward but a proof can be found in [Stevenson 2013, Lemma 3.8]). It is immediate from the definition that $k(p) \in \mathbf{M}$ and so $\langle k(p)\rangle \subseteq \mathrm{M}$. By Neeman's classification result [1992] we have $\Gamma_{p} R \in\langle k(p)\rangle$, and hence $\Gamma_{p} R$ also lies in M. Thus $\Gamma_{p} R \otimes_{R} X \in\left\langle k(p) \otimes_{R} X\right\rangle$ and it only remains to observe that $X \in \Gamma_{p} \mathrm{D}(R C)$ implies $\Gamma_{p} R \otimes_{R} X \simeq X$.

Corollary 4.3. Let $R$ be a commutative noetherian ring and $C$ a small category. Then the assignments

$$
\{\text { localizing subcategories } \mathrm{L} \text { of } \mathrm{D}(R C)\} \stackrel{f}{\rightleftarrows}\{\text { sections l of } \mathcal{L} \stackrel{s}{\longrightarrow} \operatorname{Spec} R\}
$$

are inverse to one another.

Proof. It is sufficient to verify condition (3) of Proposition 4.1, i.e., that for every $X \in \Gamma_{p} \mathrm{D}(R C)$ we have $X \in\left\langle k(p) \otimes_{R} X\right\rangle$. This is precisely the content of the theorem and so we see that $f$ and $g$ are inverse.

Remark 4.4. As noted in Remark 2.6, our results are also valid in the case where $D$ is a flat $R$-linear category and we consider $\mathrm{D}\left(\operatorname{Mod}_{R} D\right)$. One just needs to replace $k(p) C$ by $k(p) \otimes_{R} D$, the base change of $D$ to $k(p)$; the arguments don't change.

\section{Dynkin quivers}

In this section we give a concrete application of the formalism above by considering the case where $C$ is the path category of a simply laced Dynkin quiver. Let $Q$ be a quiver whose underlying graph is a simply laced Dynkin diagram. We can naturally view $Q$ as a poset, i.e., a small category, and apply our result to the study of the derived category, $\mathrm{D}(R Q)$, of representations of $Q$ over $R$. This yields the following extension of work of Ingalls and Thomas [2009], where we refer the reader for information about noncrossing partitions.

Corollary 5.1. Let $R$ be a commutative noetherian ring and $Q$ a simply laced Dynkin quiver, and denote by $R Q$ the $R$-linear path algebra of $Q$. There is an isomorphism of lattices

$$
\{\text { localizing subcategories of } \mathrm{D}(R Q)\} \underset{g}{\stackrel{f}{\rightleftarrows}}\{\text { functions } \operatorname{Spec} R \rightarrow \mathrm{NC}(Q)\} \text {, }
$$

where $\mathrm{NC}(Q)$ denotes the lattice of noncrossing partitions associated to $Q$.

Proof. Corollary 4.3 applies so it just remains to demonstrate that there is a bijection $\{$ sections of $\mathcal{L} \stackrel{s}{\longrightarrow} \operatorname{Spec} R\} \simeq \operatorname{Hom}(\operatorname{Spec} R, \operatorname{NC}(Q))$. 
This follows from [Krause 2012, Theorem 6.10] which shows, without restriction on the field $k$, that there is a bijection between the lattice of thick subcategories of $\mathrm{D}^{b}(k Q)$ and $\mathrm{NC}(Q)$. As $k Q$ is hereditary and of finite representation type, $\mathrm{D}(k Q)$ is pure-semisimple, i.e., every object is a direct sum of compact objects, and so we deduce a bijection between the lattice of localizing subcategories of $\mathrm{D}(k Q)$ and $\mathrm{NC}(Q)$. Thus sections of $\mathcal{L} \rightarrow \operatorname{Spec} R$ are nothing but functions from $\operatorname{Spec} R$ to $\operatorname{NC}(Q)$.

Remark 5.2. One can also use Lemma 3.10 and Krause's extension [2012, Theorem 6.10] of a result by Igusa and Schiffler to get partial information on the lattice of localizing subcategories of $\mathrm{D}(R Q)$ for an arbitrary quiver $Q$.

In this situation we can obtain a classification of the thick subcategories of $\mathrm{D}^{\text {perf }}(R Q)$, the category of perfect complexes of $R Q$-modules. Recall that $\mathrm{D}^{\text {perf }}(R Q)$ is the full subcategory of $\mathrm{D}(R Q)$ consisting of those objects quasi-isomorphic to a bounded complex of finitely generated projective modules; it is a thick subcategory and is the subcategory of compact objects in $\mathrm{D}(R Q)$. As in the case of $\mathrm{D}^{\text {perf }}(R)$, the thick subcategories of $\mathrm{D}^{\text {perf }}(R Q)$ are given by a sublattice of the lattice of localizing subcategories defined by a certain specialization closure condition.

Definition 5.3. We call a function $\sigma: \operatorname{Spec} R \rightarrow \mathrm{NC}(Q)$ specialization closed if whenever $p \subseteq q$ we have $\sigma(p) \leq \sigma(q)$ in $\mathrm{NC}(Q)$.

Remark 5.4. This recovers the usual notion of specialization closure of subsets of Spec $R$ when $Q=A_{1}$ and so $\operatorname{NC}(Q)=\{0,1\}$. Moreover, returning to the general simply laced case, if $\mathrm{L}$ is a localizing subcategory with $f(\mathrm{~L})$ specialization closed then for $p \subseteq q$ we have

$$
k(p) \otimes \mathrm{L} \neq 0 \quad \Rightarrow \quad k(q) \otimes \mathrm{L} \neq 0 .
$$

We will show that specialization closed functions $\operatorname{Spec} R \rightarrow \mathrm{NC}(Q)$ classify smashing subcategories of $\mathrm{D}(R Q)$ and that the telescope conjecture holds. Combining these two results gives the claimed classification result for thick subcategories of $\mathrm{D}^{\text {perf }}(R Q)$. We begin by recalling a useful fact and then present the easiest part of the argument.

Lemma 5.5. Let $p$ be a prime ideal of $R$ and let $M$ be an indecomposable $k(p) Q$ module with dimension vector $\alpha$. Then there is a rigid lattice $\tilde{M}$ over $R Q$, i.e., $\widetilde{M}$ is $R$-free and $\operatorname{Ext}_{R Q}^{1}(\underset{\widetilde{M}}{\tilde{M}}, \tilde{M})=0$, with rank vector $\alpha$. Moreover, for any $q \in \operatorname{Spec} R$ the module $k(q) \otimes \widetilde{M}$ is the unique indecomposable $k(q) Q$-module with dimension vector $\alpha$. In particular,

$$
k(p) \otimes \tilde{M} \cong M .
$$

Proof. This is a (very) special case of [Crawley-Boevey 1996, Theorem 1].

Lemma 5.6. Let $\sigma: \operatorname{Spec} R \rightarrow \mathrm{NC}(Q)$ be specialization closed. Then the localizing subcategory $\mathrm{L}=g(\sigma)$ is generated by objects of $\mathrm{D}^{\text {perf }}(R Q)$. 
Proof. We prove this by just writing down a (rather redundant) generating set for L. For each prime ideal $p$ such that $k(p) \otimes \mathrm{L} \neq 0$, let $M(p)$ be a compact generator for the localizing subcategory of $\mathrm{D}(k(p) Q)$ generated by $k(p) \otimes \mathrm{L}$. Since $M(p)$ is a finite sum of (suspensions of) indecomposable modules in $\mathrm{D}(k(p) Q)$, we can lift it to a lattice $\widetilde{M(p)}$ in $\mathrm{D}(R Q)$ using Lemma 5.5. In particular, it is easily seen that $\widetilde{M(p)}$ is compact in $\mathrm{D}(R Q)$. Set

$$
G=\{K(p) \otimes \widetilde{M(p)} \mid p \in \operatorname{Spec} R \text { with } k(p) \otimes \mathrm{L} \neq 0\} \quad \text { and } \quad \mathrm{L}^{\prime}=\langle G\rangle,
$$

where $K(p)$ denotes the Koszul complex for $p$ defined by

$$
K(p)=\bigotimes_{i=1}^{r} \operatorname{cone}\left(R \stackrel{f_{i}}{\longrightarrow} R\right),
$$

where $p$ is generated by $f_{1}, \ldots, f_{r}$. (Recall that this implicitly means the derived tensor product over $R$.) Since $K(p) \in \mathrm{D}^{\text {perf }}(R)$ and $\widetilde{M(p)} \in \mathrm{D}^{\text {perf }}(R Q)$, the set $G$ consists of compact objects by [Stevenson 2013, Lemma 4.6].

For primes $p \subseteq q \in \operatorname{Spec} R$ the object $k(q) \otimes(K(p) \otimes \widetilde{M(p)})$ is a finite sum of suspensions of copies of the $k(q) Q$-module $k(q) \otimes \widetilde{M(p)}$. This latter module can be described as follows: each indecomposable summand of $M(p)$ corresponds to an indecomposable $k(q) Q$-module, namely the indecomposable $k(q) Q$-module with the same dimension vector, and $k(q) \otimes \widetilde{M(p)}$ is the corresponding sum of these indecomposable $k(q) Q$-modules. In particular, $M(p)$ and $k(q) \otimes \widetilde{M(p)}$ correspond to the same element of $\mathrm{NC}(Q)$. If, on the other hand, $p \nsubseteq q$ then $k(q) \otimes(K(p) \otimes \widetilde{M(p)})=0$.

Putting everything together we see that

$$
\begin{aligned}
\left\langle k(q) \otimes \mathrm{L}^{\prime}\right\rangle & =\langle k(q) \otimes K(p) \otimes \widetilde{M(p)}| p \in \operatorname{Spec} R \text { with } k(p) \otimes \mathrm{L} \neq 0\rangle \\
& =\langle k(q) \otimes \widetilde{M(q)}\rangle=\langle M(q)\rangle=\langle k(q) \otimes \mathrm{L}\rangle,
\end{aligned}
$$

where the second equality follows from the computation in the preceding paragraph together with specialization closure of $\sigma$, and the third and fourth equalities are by definition of $M(q)$ and $\widetilde{M(q)}$. This shows that $f(\mathrm{~L})=f\left(\mathrm{~L}^{\prime}\right)$ and thus, by the classification of localizing subcategories, $\mathrm{L}=\mathrm{L}^{\prime}$. We have thus exhibited a set of generators $G \subseteq \mathrm{D}^{\text {perf }}(R Q)$ for L.

We now continue with proving that the specialization closed functions $\operatorname{Spec} R \rightarrow$ $\mathrm{NC}(Q)$ classify smashing subcategories of $\mathrm{D}(R Q)$. Combined with the above lemma, this proves the telescope conjecture and classifies the thick subcategories of $\mathrm{D}^{\text {perf }}(R Q)$.

Fix a smashing subcategory $\mathrm{S}$ of $\mathrm{D}(R Q)$, i.e., consider a localization sequence

$$
\mathrm{S} \underset{i^{!}}{\stackrel{i_{*}}{\rightleftarrows}} \mathrm{D}(R Q) \underset{j_{*}}{\stackrel{j^{*}}{\rightleftarrows}} \mathrm{S}^{\perp},
$$


where $i^{!}$and $j_{*}$ are the right adjoints of the inclusion functors $i_{*}$ and the localization functor $j^{*}$, respectively, and all of these functors preserve coproducts. In particular, $\mathrm{S}^{\perp}$ is also a localizing subcategory of $\mathrm{D}(R Q)$. In order to prove the result indicated above we start with two elementary lemmas.

Lemma 5.7. Let $\mathrm{S}$ be as above. Then for any $Y \in \mathrm{D}(R)$ and $X \in \mathrm{D}(R Q)$ we have canonical isomorphisms

$$
i_{*} i^{!}(Y \otimes X) \cong Y \otimes i_{*} i^{!} X \text { and } j_{*} j^{*}(Y \otimes X) \cong Y \otimes j_{*} j^{*} X .
$$

Proof. Consider the localization triangle for $X$

$$
i_{*} i^{!} X \rightarrow X \rightarrow j_{*} j^{*} X \rightarrow \Sigma i_{*} i^{!} X .
$$

Acting on this triangle with $Y$ gives a new triangle

$$
Y \otimes i_{*} i^{!} X \rightarrow Y \otimes X \rightarrow Y \otimes j_{*} j^{*} X \rightarrow \Sigma\left(Y \otimes i_{*} i^{!} X\right) .
$$

By Lemma 3.3 both $\mathrm{S}$ and $\mathrm{S}^{\perp}$ are closed under the $\mathrm{D}(R)$ action and so we have $Y \otimes i_{*} i^{!} X \in \mathrm{S}$ and $Y \otimes j_{*} j^{*} X \in \mathrm{S}^{\perp}$. The claimed isomorphisms follow immediately from the uniqueness of localization triangles.

Lemma 5.8. Let $p^{\prime} \in \operatorname{Spec} R$. Let $M, N$ be indecomposable $k\left(p^{\prime}\right) Q$-modules with

$$
\operatorname{Hom}_{k\left(p^{\prime}\right) Q}(M, N) \neq 0
$$

and denote choices of their respective rigid lattice lifts by $\widetilde{M}$ and $\widetilde{N}$. Then, given $p \subseteq q \in \operatorname{Spec} R$, we have

$$
\operatorname{Hom}_{R Q}(E(k(p)) \otimes \tilde{M}, E(k(q)) \otimes \widetilde{N}) \neq 0,
$$

where $E(k(p)), E(k(q))$ denote the injective envelopes of the residue fields $k(p), k(q)$. Proof. We know there are rigid lattice lifts of $M$ and $N$ by Lemma 5.5. We can choose, using the classification of indecomposable modules over $Q$, a nonzero $\phi: M \rightarrow N$ given on each component by matrices involving only zero and identity maps. It is then clear that we can lift it to a nonzero $\tilde{\phi}: \widetilde{M} \rightarrow \widetilde{N}$ such that $\tilde{\phi}$, like $\phi$, is given componentwise by matrices whose only entries are zero and identity maps. On the other hand, since $p \subseteq q$, there is a nonzero map $\psi: E(k(p)) \rightarrow E(k(q))$. It is thus evident by our choice of $\tilde{\phi}$ that either of the equal composites in the commutative square

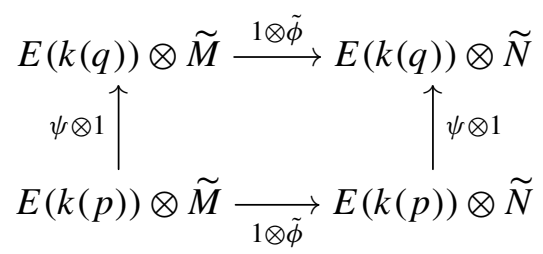

gives the desired nonzero morphism. 
Using this series of easy observations we can now dispose of the proof of the theorem in short order.

Theorem 5.9. Let $\mathrm{S}$ be a smashing subcategory of $\mathrm{D}(R Q)$ with notation as introduced above. Then $f(\mathrm{~S}): \operatorname{Spec} R \rightarrow \mathrm{NC}(Q)$ is specialization closed.

Proof. Fix $p \subseteq q \in$ Spec $R$ and an indecomposable module $M \in k(p) \otimes \mathrm{S} \subseteq \mathrm{D}(k(p) Q)$ with dimension vector $\alpha$. By Lemma 5.5 there is a lattice $\tilde{M} \in \mathrm{D}^{\text {perf }}(R Q)$ with $k(p) \otimes \tilde{M} \cong M$ and $k(q) \otimes \widetilde{M}$ the unique indecomposable $k(q) Q$-module with dimension vector $\alpha$. We have to show that $k(q) \otimes \widetilde{M}$ is in $k(q) \otimes \mathrm{S}$. To this end consider the localization triangle

$$
i_{*} i^{!} \tilde{M} \rightarrow \tilde{M} \rightarrow j_{*} j^{*} \tilde{M} \rightarrow \Sigma i_{*} i^{!} \tilde{M} .
$$

Pick an indecomposable summand $N$ of $k(q) \otimes j_{*} j^{*} \widetilde{M}$ and note that, by Lemma 5.7, $N \in \mathrm{S}^{\perp}$. We assume $N$ is nonzero since if $k(q) \otimes j_{*} j^{*} \tilde{M}$ is zero then $k(q) \otimes \tilde{M}$ is in $\mathrm{S}$ and we are done. Let $\tilde{N}$ be a lattice lift of $N$. As we have assumed $k(q) \otimes j_{*} j^{*} \widetilde{M}$ is nonzero, the morphism

$$
\phi=k(q) \otimes \tilde{M} \rightarrow k(q) \otimes j_{*} j^{*} \widetilde{M} \rightarrow N \cong k(q) \otimes \widetilde{N}
$$

must also be nonzero. Thus we can apply Lemma 5.8 to produce a nonzero morphism

$$
\gamma: E(k(p)) \otimes \tilde{M} \rightarrow E(k(q)) \otimes \widetilde{N}
$$

in $\mathrm{D}(R Q)$.

On the other hand, by assumption $k(p) \otimes \tilde{M} \in \mathrm{S}$ and $k(q) \otimes \widetilde{N} \in \mathrm{S}^{\perp}$. Since both $\mathrm{S}$ and $\mathrm{S}^{\perp}$ are localizing, and since for any prime ideal $p^{\prime}$ we have $E\left(k\left(p^{\prime}\right)\right) \in\left\langle k\left(p^{\prime}\right)\right\rangle$, we see (as in the proof of Theorem 4.2) that

$$
E(k(p)) \otimes \widetilde{M} \in \mathrm{S} \quad \text { and } \quad E(k(q)) \otimes \widetilde{N} \in \mathrm{S}^{\perp} .
$$

But this contradicts the existence of the nonzero morphism $\gamma$. Hence $N$ must have been zero, showing that $k(q) \otimes j_{*} j^{*} \tilde{M} \cong 0$, which in turn implies (via Lemma 5.7) that $k(q) \otimes \tilde{M} \in \mathrm{S}$ as desired.

This theorem has the following, more palatable, consequences.

Corollary 5.10. Let $R$ be a commutative noetherian ring and $Q$ a simply laced Dynkin quiver. Then $\mathrm{D}(R Q)$ satisfies the telescope conjecture: every smashing subcategory is generated by objects of $\mathrm{D}^{\text {perf }}(R Q)$.

Proof. Suppose $\mathrm{S}$ is a smashing subcategory. Then by the classification given in Corollary 5.1 we know $\mathrm{S}=g f(\mathrm{~S})$. By Theorem 5.9 the function $f(\mathrm{~S})$ is specialization closed and so by Lemma 5.6 we see that $\mathrm{S}=g f(\mathrm{~S})$ is generated by objects of $\mathrm{D}^{\text {perf }}(R Q)$ as claimed. 


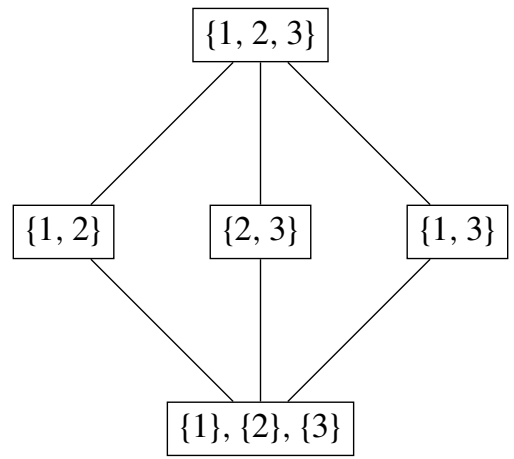

Figure 1. The lattice of noncrossing partitions of $\{1,2,3\}$. The coarser partitions are decreed to be bigger in the lattice structure.

Corollary 5.11. Let $R$ be a commutative noetherian ring and $Q$ a simply laced Dynkin quiver. There is an isomorphism of lattices

$$
\left\{\begin{array}{c}
\text { thick subcategories } \\
\text { of } \mathrm{D}^{\mathrm{perf}}(R Q)
\end{array}\right\} \stackrel{f}{\rightleftarrows}\left\{\begin{array}{c}
\text { specialization closed functions } \\
\operatorname{Spec} R \rightarrow \mathrm{NC}(Q)
\end{array}\right\}
$$

where $\mathrm{NC}(Q)$ denotes the lattice of noncrossing partitions associated to $Q$.

Proof. Considering the classification of Corollary 5.1 and combining Theorem 5.9 and Lemma 5.6 gives a classification of the smashing subcategories of $\mathrm{D}(R Q)$ in terms of the specialization closed functions $\operatorname{Spec} R \rightarrow \mathrm{NC}(Q)$. By the previous corollary this is also the classification of the localizing subcategories of $\mathrm{D}(R Q)$ generated by objects of $\mathrm{D}^{\text {perf }}(R Q)$. One obtains the isomorphism we have asserted in the statement in the standard way: by Thomason's localization theorem (see, for example, [Neeman 1996, Theorem 2.1]) the thick subcategories of $\mathrm{D}^{\text {perf }}(R Q)$ are in order-preserving bijection with the localizing subcategories of $\mathrm{D}(R Q)$ which are generated by perfect complexes.

Example 5.12. Let $R$ be a local 1-dimensional domain. Then Spec $R$ consists of two points: a generic point $\eta$ and a closed point $x$. We will consider the case of $Q=A_{2}$ in Corollary 5.11. The lattice $\mathrm{NC}\left(A_{2}\right)$ consists of the noncrossing partitions of the set $\{1,2,3\}$. A noncrossing partition of a cyclically ordered set $S$ determined by an equivalence relation $\sim$ is one where $x<y<z<w, x \sim z$, and $y \sim w$ together imply $x \sim y \sim z \sim w$.

In Figure 1 we display each partition as determined by its largest equivalence classes. The class of all localizing subcategories of $\mathrm{D}\left(R A_{2}\right)$ in this case is simply two copies of this lattice, indexed on $\eta$ and $x$. Figure 2 shows the lattice of specialization closed functions $\operatorname{Spec} R \rightarrow \mathrm{NC}\left(A_{2}\right)$, which by the results above is the lattice of thick subcategories of $\mathrm{D}^{\text {perf }}\left(R A_{2}\right)$. 


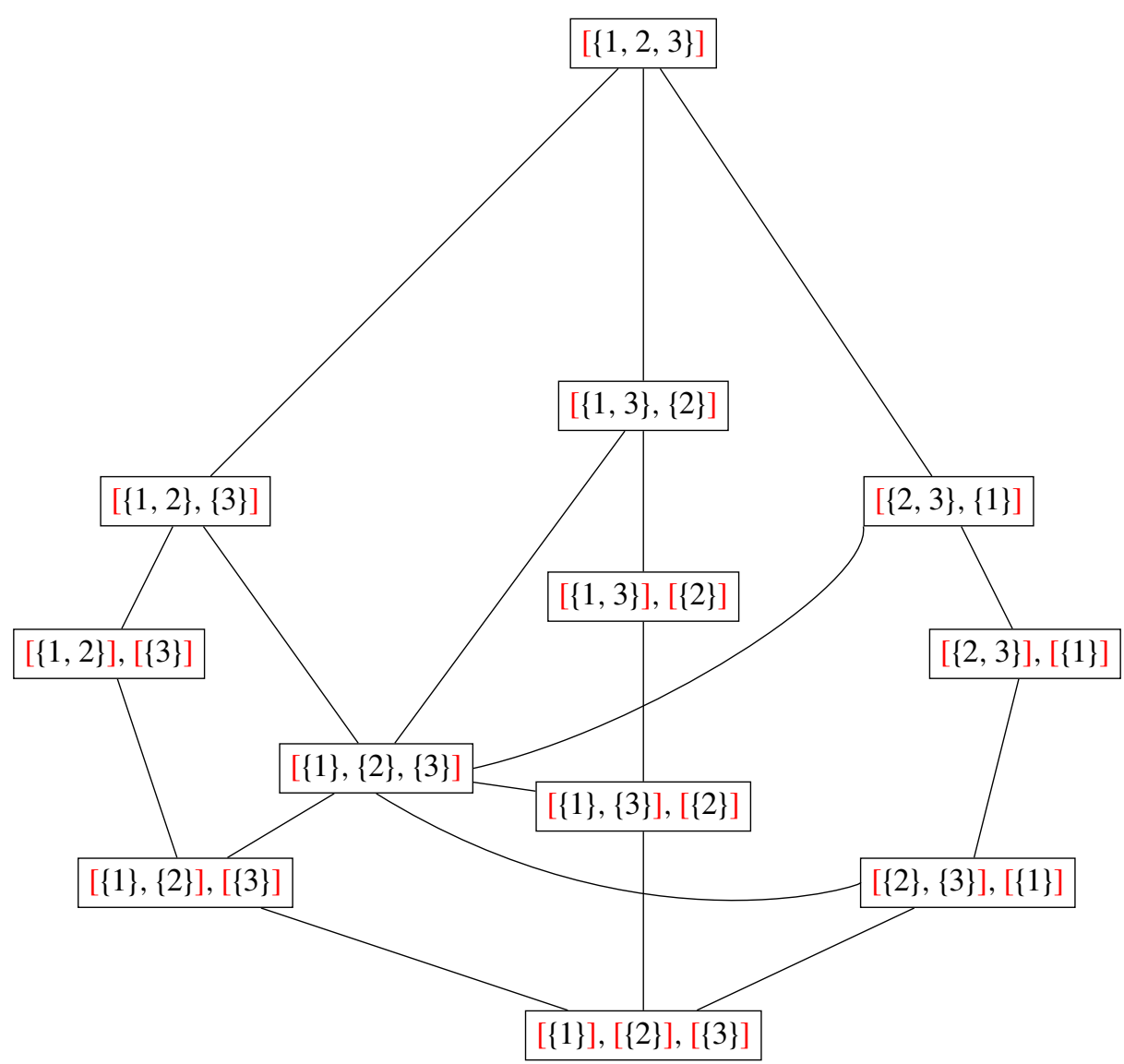

Figure 2. The lattice of specialization closed functions $\operatorname{Spec} R \rightarrow$ $\mathrm{NC}\left(A_{2}\right)$ for $R$ a 1-dimensional local domain. The partition given by the black parentheses is the noncrossing partition corresponding to the generic point $\eta$, while the partition determined by the red parentheses is the partition corresponding to the closed point $x$.

\section{Towards telescopy}

We have seen in Corollary 5.10 that the telescope conjecture holds for $\mathrm{D}(R Q)$ when $Q$ is an $\mathrm{ADE}$ quiver and $R$ is any commutative noetherian ring. Unfortunately we were not able to prove such a general statement for even arbitrary quivers, let alone arbitrary small categories. However, we do have some partial results and remarks that we present in this section which revolve around the following question.

Question 6.1. Let $R$ be a noetherian commutative ring. Does the telescope conjecture hold for $\mathrm{D}(R C)$ when $C$ is an ordinary (not $R$-linear) category if it holds for $\mathrm{D}(k(p) C)$ for all $p \in \operatorname{Spec} R$ ? 
We begin to answer this question by showing that the bijection of Proposition 4.1(2) restricts to a bijection between the collections of smashing subcategories. Given a localizing subcategory L of some triangulated category, we will denote the associated acyclization and localization functors by $\Gamma_{\mathrm{L}}$ and $L_{\mathrm{L}}$ respectively.

Remark 6.2. Throughout we will prove that some localizing subcategory $S$ is smashing by exhibiting that the right orthogonal $S^{\perp}$ is also localizing. In order for this condition to be equivalent to $S$ being smashing, one needs to know that the inclusion of $S$ admits a right adjoint. In all of the cases we consider $S$ will clearly be generated by a set of objects; for instance, it will be the localizing subcategory generated by the image of some other smashing subcategory under an exact functor, and so the existence of the adjoint follows from Brown representability. Indeed, in this case one has a generating set, as any smashing subcategory of a compactly generated triangulated category has a set of generators by [Krause 2010, Theorem 7.4.1], and so one can apply Brown representability for well-generated categories as in [Neeman 2001] (or see [Krause 2010, Theorem 5.1.1]). Thus we will suppress this part of the arguments throughout.

For the moment, fix some $p \in \operatorname{Spec} R$ and denote by $i^{*}$ the functor $k(p) \otimes(-)$ : $\Gamma_{p} \mathrm{D}(R C) \rightarrow \mathrm{D}(k(p) C)$ and by $i_{*}$ its right adjoint.

Lemma 6.3. Suppose $\mathrm{S}$ is a smashing subcategory of $\Gamma_{p} \mathrm{D}(R C)$ and set

$$
\mathrm{T}=f(\mathrm{~S})=\operatorname{add}(k(p) \otimes \mathrm{S}) \quad \text { and } \quad \mathrm{T}^{\prime}=f\left(\mathrm{~S}^{\perp}\right)=\operatorname{add}\left(k(p) \otimes \mathrm{S}^{\perp}\right) .
$$

Then $\mathrm{T}^{\prime}$ is the right orthogonal of $\mathrm{T}$, and hence $\mathrm{T}$ is a smashing subcategory of $\mathrm{D}(k(p) C)$.

Proof. If $X \in \mathrm{T}^{\prime}$ then there is, by definition, some $\bar{X} \in \mathrm{S}^{\perp}$ such that $X$ is a summand of $i^{*} \bar{X}$. Given $Y \in \mathrm{T}$, which we can assume to be of the form $i^{*} \bar{Y}$ with $\bar{Y} \in \mathrm{S}$, we have

$$
\operatorname{Hom}\left(i^{*} \bar{Y}, i^{*} \bar{X}\right) \cong \operatorname{Hom}\left(\bar{Y}, i_{*} i^{*} \bar{X}\right) .
$$

This latter hom-set is zero, as $\bar{Y} \in \mathrm{S}$ and $i_{*} i^{*} \bar{X} \in \mathrm{S}^{\perp}$ by the closure of localizing subcategories under the $\mathrm{D}(R)$ action. Thus $\mathrm{T}^{\prime} \subseteq \mathrm{T}^{\perp}$.

On the other hand, if $\operatorname{Hom}\left(i^{*} \mathrm{~S}, Z\right)=0$ for some $Z \in \mathrm{D}(k(p) C)$, then by adjunction $i_{*} Z \in \mathrm{S}^{\perp}$. Hence $i^{*} i_{*} Z \in \mathrm{T}^{\prime}$ and we know, by Lemma 3.7, that $Z$ is a summand of $i^{*} i_{*} Z$. So $Z$ is in $\mathrm{T}^{\prime}$, proving that $\mathrm{T}^{\perp} \subset \mathrm{T}^{\prime}$ and completing the argument.

Now we fix a smashing subcategory $\mathrm{T}$ of $\mathrm{D}(k(p) C)$ and set

$$
\mathrm{S}=g(\mathrm{~T})=\left\langle i_{*} \mathrm{~T}\right\rangle \quad \text { and } \quad \mathrm{S}^{\prime}=g\left(\mathrm{~T}^{\perp}\right)=\left\langle i_{*} \mathrm{~T}^{\perp}\right\rangle .
$$

We wish to show that $S$ is smashing with right orthogonal $S^{\prime}$. We prove this in the following four statements. 
Lemma 6.4. The subcategories $\mathrm{S}$ and $\mathrm{S}^{\prime}$ generate $\Gamma_{p} \mathrm{D}(R C)$, i.e., we have

$$
\left\langle\mathrm{S} \cup \mathrm{S}^{\prime}\right\rangle=\Gamma_{p} \mathrm{D}(R C) .
$$

Proof. Let $X$ be an object of $\Gamma_{p} \mathrm{D}(R C)$. By Theorem 4.2 we know $X$ is in the localizing subcategory $\left\langle i_{*} i^{*} X\right\rangle$. We have a localization triangle in $\mathrm{D}(k(p) C)$

$$
\Gamma_{\mathrm{T}} i^{*} X \rightarrow i^{*} X \rightarrow L_{\mathrm{T}} i^{*} X \rightarrow \Sigma \Gamma_{\mathrm{T}} i^{*} X
$$

where $\Gamma_{\mathrm{T}} i^{*} X \in \mathrm{T}$ and $L_{\mathrm{T}} i^{*} X \in \mathrm{T}^{\perp}$. Applying $i_{*}$ gives a triangle in $\mathrm{D}(R C)$

$$
i_{*} \Gamma_{\mathrm{T}} i^{*} X \rightarrow i_{*} i^{*} X \rightarrow i_{*} L_{\mathrm{T}} i^{*} X \rightarrow \Sigma i_{*} \Gamma_{T} i^{*} X
$$

with $i_{*} \Gamma_{\mathrm{T}} i^{*} X \in \mathrm{S}$ and $i_{*} L_{\mathrm{T}} i^{*} X \in \mathrm{S}^{\prime}$ by definition. Thus $X \in\left\langle i_{*} i^{*} X\right\rangle \subseteq\left\langle\mathrm{S} \cup \mathrm{S}^{\prime}\right\rangle$, as claimed.

Lemma 6.5. There is a containment of triangulated subcategories $\mathrm{S}^{\prime} \subseteq \mathrm{S}^{\perp}$.

Proof. It is enough to check that for every $t \in \mathrm{T}$ and $t^{\prime} \in \mathrm{T}^{\perp}$ we have

$$
\operatorname{Hom}\left(i_{*} t, i_{*} t^{\prime}\right)=0 \text {. }
$$

The required vanishing follows from the isomorphisms

$$
\operatorname{Hom}\left(i_{*} t, i_{*} t^{\prime}\right) \cong \operatorname{Hom}\left(i^{*} i_{*} t, t^{\prime}\right) \cong \operatorname{Hom}\left(\coprod_{\lambda} \Sigma^{n_{\lambda}} t, t^{\prime}\right) \cong \prod_{\lambda} \operatorname{Hom}\left(\Sigma^{n_{\lambda}} t, t^{\prime}\right)=0,
$$

where the first isomorphism is by adjunction, the second is by Lemma 3.7, and the final hom-set vanishes by assumption.

Lemma 6.6. There is an equality

$\Gamma_{p} \mathrm{D}(R C)=\left\{X \in \Gamma_{p} \mathrm{D}(R C) \mid\right.$ there exists a triangle $X^{\prime} \rightarrow X \rightarrow X^{\prime \prime} \rightarrow \Sigma X^{\prime}$ with $X^{\prime} \in \mathrm{S}$ and $\left.X^{\prime \prime} \in \mathrm{S}^{\prime}\right\}$.

Proof. It is routine to verify that the full subcategory defined on the right-hand side above is localizing, and it contains $S$ and $S^{\prime}$ by definition. The equality then follows from Lemma 6.4 .

Proposition 6.7. $\mathrm{S}$ is smashing in $\Gamma_{p} \mathrm{D}(R C)$ with right orthogonal $\mathrm{S}^{\prime}$.

Proof. We already know by Lemma 6.5 that $S^{\prime} \subseteq S^{\perp}$. Let $X$ be an object of $S^{\perp}$. By the last lemma we know there is a triangle

$$
X^{\prime} \rightarrow X \rightarrow X^{\prime \prime} \rightarrow \Sigma X^{\prime}
$$

with $X^{\prime} \in \mathrm{S}$ and $X^{\prime \prime} \in \mathrm{S}^{\prime}$. But, since $X \in \mathrm{S}^{\perp}$, the first map must vanish, implying $X^{\prime \prime} \cong X \oplus \Sigma X^{\prime}$. This in turn implies $X^{\prime} \cong 0$ since $\mathrm{S} \cap \mathrm{S}^{\prime}=0$. We thus conclude that $X \cong X^{\prime \prime}$, i.e., $X \in \mathrm{S}^{\prime}$, proving $\mathrm{S}^{\perp}=\mathrm{S}^{\prime}$. In particular, $\mathrm{S}$ is smashing. 
We now have enough to prove that we can describe the smashing subcategories of $\Gamma_{p} \mathrm{D}(R C)$ in terms of the smashing subcategories of $\mathrm{D}(k(p) C)$.

Theorem 6.8. There is an order-preserving bijection

$$
\left\{\begin{array}{c}
\text { smashing subcategories } \\
\text { of } \Gamma_{p} \mathrm{D}(R C)
\end{array}\right\} \stackrel{f_{p}}{\underset{g_{p}}{\rightleftarrows}}\left\{\begin{array}{c}
\text { smashing subcategories } \\
\text { of } \mathrm{D}(k(p) C)
\end{array}\right\} \text {. }
$$

Proof. We know from Proposition 4.1(2) that there is a bijection between the sets of localizing subcategories of $\Gamma_{p} \mathrm{D}(R C)$ and $\mathrm{D}(k(p) C)$ given by $f_{p}$ and $g_{p}$. By Lemma 6.3 and Proposition 6.7 both $f_{p}$ and $g_{p}$ send smashing subcategories to smashing subcategories and so the bijection restricts as claimed.

Obtaining the corresponding result for localizing subcategories generated by compact objects of $\Gamma_{p} \mathrm{D}(R C)$ and $\mathrm{D}(k(p) C)$ seems more subtle. However, if $R$ is sufficiently nice at the prime ideal $p$ this is possible. In order to state the result we need a simple preparatory lemma.

Lemma 6.9. Let $p$ be a prime ideal of $\operatorname{Spec} R$. The category $\Gamma_{p} \mathrm{D}(R C)$ is a compactly generated triangulated category.

Proof. Recall that $\Gamma_{p} \mathrm{D}(R C)$ is the essential image of acting by

$$
\Gamma_{p} R=K_{\infty}(p) \otimes_{R} R_{p}
$$

It is clear that $\mathrm{D}\left(R_{p} C\right)$, the essential image of acting by $R_{p}$, is a compactly generated triangulated category. By [Stevenson 2013, Corollary 4.11] the essential image of $K_{\infty}(p)_{p} \otimes_{R_{p}}(-)$ acting on $\mathrm{D}\left(R_{p} C\right)$, namely $\Gamma_{p} \mathrm{D}(R C)$, is also compactly generated (even by objects of $\mathrm{D}^{\text {perf }}\left(R_{p} C\right)$ ).

In the statement and proof of the following proposition, $\left(\Gamma_{p} \mathrm{D}(R C)\right)^{c}$ denotes the full subcategory of compact objects of $\Gamma_{p} \mathrm{D}(R C)$.

Proposition 6.10. Let $p$ be a prime ideal of $R$ such that $R_{p}$ is regular. Then the assignments $f_{p}$ and $g_{p}$ of Proposition 4.1(2) induce an order-preserving bijection between localizing subcategories of $\Gamma_{p} \mathrm{D}(R C)$ generated by objects of $\left(\Gamma_{p} \mathrm{D}(R C)\right)^{c}$ and localizing subcategories of $\mathrm{D}(k(p) C)$ generated by objects of $\mathrm{D}^{\text {perf }}(k(p) C)$.

Proof. The base change functor $\Gamma_{p} \mathrm{D}(R C) \rightarrow \mathrm{D}(k(p) C)$ has a coproduct-preserving right adjoint and so sends compacts to compacts by [Neeman 1996, Theorem 5.1]. Thus it is clear that $f_{p}$ sends any localizing subcategory of $\Gamma_{p} \mathrm{D}(R C)$ generated by objects of $\left(\Gamma_{p} \mathrm{D}(R C)\right)^{c}$ to a localizing subcategory generated by objects of $\mathrm{D}^{\text {perf }}(k(p) C)$. The argument for $g_{p}$ is similar, using the fact that, as $R_{p}$ is regular, the residue field $k(p)$ is compact, and so the right adjoint of the restriction functor $\operatorname{Hom}_{R}(k(p),-)$ is also coproduct-preserving. 
As an immediate consequence of the theorem and the proposition we deduce the following corollary.

Corollary 6.11. Suppose $R_{p}$ is regular. Then $\Gamma_{p} \mathrm{D}(R C)$ satisfies the telescope conjecture if and only if $\mathrm{D}(k(p) C)$ satisfies the telescope conjecture.

Proof. Suppose $\mathrm{D}(k(p) C)$ satisfies the telescope conjecture and let $\mathrm{S}$ be a smashing subcategory of $\Gamma_{p} \mathrm{D}(R C)$. Then $f_{p}(S)$ is smashing in $\mathrm{D}(k(p) C)$ by Theorem 6.8 and we have $g_{p} f_{p}(\mathrm{~S})=\mathrm{S}$. Since we have assumed the telescope conjecture for $\mathrm{D}(k(p) C)$, we know $f_{p}(S)$ is generated by objects of $\mathrm{D}^{\text {perf }}(k(p) C)$. Applying Proposition 6.10 we deduce that $\mathrm{S}=g_{p} f_{p}(\mathrm{~S})$ is generated by objects which are compact in $\Gamma_{p} \mathrm{D}(R C)$. Thus the telescope conjecture holds for $\Gamma_{p} \mathrm{D}(R C)$. The other implication is clear since $i^{*}$ preserves compact objects.

This corollary already buys us something in a concrete setting, although it is not clear how to extend it to all of $\mathrm{D}(R C)$.

Corollary 6.12. Let $Q$ be a quiver and let $R$ be a commutative noetherian ring. For each $p \in \operatorname{Spec} R$ such that $R_{p}$ is regular, the telescope conjecture holds for $\Gamma_{p} \mathrm{D}(R C)$.

Proof. By the previous corollary it is sufficient to verify the telescope conjecture for $\mathrm{D}(k(p) Q)$. This has been done by Krause and Štovíček [2010, Theorem 7.1].

We give one additional lemma that could prove useful in resolving Question 6.1.

Lemma 6.13. If $\mathrm{S}$ is a smashing subcategory of $\mathrm{D}(R C)$ then for every $p \in \operatorname{Spec} R$ the localizing subcategory $\Gamma_{p} \mathrm{~S}$ is smashing in $\Gamma_{p} \mathrm{D}(R C)$.

Proof. It is not hard to check that both $\Gamma_{p} \mathrm{~S}$ and $\Gamma_{p}\left(\mathrm{~S}^{\perp}\right)$ are localizing subcategories of $\Gamma_{p} \mathrm{D}(R C)$. Moreover,

$$
\Gamma_{p} \mathrm{~S} \subseteq \mathrm{S} \quad \text { and } \quad \Gamma_{p}\left(\mathrm{~S}^{\perp}\right) \subseteq \mathrm{S}^{\perp}
$$

by Lemma 3.3. In particular, $\Gamma_{p}\left(\mathrm{~S}^{\perp}\right) \subseteq\left(\Gamma_{p} \mathrm{~S}\right)^{\perp}$. Applying $\Gamma_{p} R \otimes_{R}(-)$ to localization triangles for $\mathrm{S}$ shows that every object $X$ of $\Gamma_{p} \mathrm{D}(R C)$ fits into a triangle

$$
X^{\prime} \rightarrow X \rightarrow X^{\prime \prime} \rightarrow \Sigma X^{\prime}
$$

with $X^{\prime} \in \Gamma_{p} S$ and $X^{\prime \prime} \in \Gamma_{p}\left(\mathrm{~S}^{\perp}\right)$. One can conclude the proof by arguing as in the proof of Proposition 6.7.

In summary, we understand what happens at "points" and we can pass from a smashing subcategory of $\mathrm{D}(R C)$ to a smashing subcategory at each prime. What is not clear is how to use this pointwise information to deduce something about the original smashing subcategory. The naive idea, based on the existing proofs of the telescope conjecture in various instances, would be to prove some sort of specialization closure condition for the section corresponding to a smashing 
subcategory as in Theorem 5.9. One could then hope to combine such a condition with the fiberwise results above. However, the following example shows that one cannot always expect specialization closure.

Example 6.14. Consider the projection Spec $k[x, y] \rightarrow \operatorname{Spec} k[x]$. We then view $\operatorname{Mod} k[x, y]$ as a $k[x]$-linear category. This gives rise to an action of $\mathrm{D}(k[x])$ on $\mathrm{D}(k[x, y])$. Let $\mathrm{S}$ be the smashing subcategory of $\mathrm{D}(k[x, y])$ determined by the closed curve $x y=1$. Then the support of $\mathrm{S}$ with respect to the action of $\mathrm{D}(k[x])$ is open in Spec $k[x]$. Of course, in this case the telescope conjecture does hold.

\section{Acknowledgements}

We would like to express our thanks to MSRI for its hospitality during the thematic program "Noncommutative algebraic geometry and representation theory" and to Universität Bielefeld for its hospitality to Antieau. We are also grateful to the anonymous referee for providing several helpful comments.

\section{References}

[Balmer 2005] P. Balmer, "The spectrum of prime ideals in tensor triangulated categories", J. Reine Angew. Math. 588 (2005), 149-168. MR 2007b:18012 Zbl 1080.18007

[Benson et al. 1997] D. J. Benson, J. F. Carlson, and J. Rickard, "Thick subcategories of the stable module category”, Fund. Math. 153:1 (1997), 59-80. MR 98g:20021 Zbl 0886.20007

[Benson et al. 2011] D. J. Benson, S. B. Iyengar, and H. Krause, "Stratifying modular representations of finite groups", Ann. of Math. (2) 174:3 (2011), 1643-1684. MR 2846489 Zbl 1261.20057

[Bousfield 1979] A. K. Bousfield, "The localization of spectra with respect to homology", Topology 18:4 (1979), 257-281. MR 80m:55006 Zbl 0417.55007

[Brüning 2007] K. Brüning, "Thick subcategories of the derived category of a hereditary algebra", Homology, Homotopy Appl. 9:2 (2007), 165-176. MR 2009d:18018 Zbl 1142.18008

[Crawley-Boevey 1996] W. Crawley-Boevey, "Rigid integral representations of quivers", pp. 155163 in Representation theory of algebras (Cocoyoc, 1994), edited by R. Bautista et al., Canadian Mathematical Society Conference Proceedings 18, American Mathematical Society, Providence, RI, 1996. MR 97e:16025 Zbl 0857.16014

[Dell' Ambrogio and Stevenson 2013] I. Dell' Ambrogio and G. Stevenson, "On the derived category of a graded commutative Noetherian ring”, J. Algebra 373 (2013), 356-376. MR 2995031 Zbl 1272.13002

[Dell'Ambrogio and Stevenson 2014] I. Dell'Ambrogio and G. Stevenson, "Even more spectra: tensor triangular comparison maps via graded commutative 2-rings", Appl. Categ. Structures 22:1 (2014), 169-210. MR 3163513 Zbl 06307128

[Devinatz et al. 1988] E. S. Devinatz, M. J. Hopkins, and J. H. Smith, "Nilpotence and stable homotopy theory I", Ann. of Math. (2) 128:2 (1988), 207-241. MR 89m:55009 Zbl 0673.55008

[Greenlees 1993] J. P. C. Greenlees, “ $K$-homology of universal spaces and local cohomology of the representation ring”, Topology 32:2 (1993), 295-308. MR 94c:19007 Zbl 0779.55005

[Hopkins and Smith 1998] M. J. Hopkins and J. H. Smith, "Nilpotence and stable homotopy theory II”, Ann. of Math. (2) 148:1 (1998), 1-49. MR 99h:55009 Zbl 0927.55015 
[Ingalls and Thomas 2009] C. Ingalls and H. Thomas, "Noncrossing partitions and representations of quivers", Compos. Math. 145:6 (2009), 1533-1562. MR 2010m:16021 Zbl 1182.16012

[Kelly 1982] G. M. Kelly, Basic concepts of enriched category theory, London Mathematical Society Lecture Note Series 64, Cambridge University Press, 1982. Corrected reprint published in Reprints in Theory and Applications of Categories 10, 2005. MR 84e:18001 Zbl 0478.18005

[Krause 2010] H. Krause, "Localization theory for triangulated categories", pp. 161-235 in Triangulated categories, edited by T. Holm et al., London Mathematical Society Lecture Note Series 375, Cambridge University Press, 2010. MR 2012e:18026 Zbl 1232.18012

[Krause 2012] H. Krause, "Report on locally finite triangulated categories”, J. K-Theory 9:3 (2012), 421-458. MR 2955969 Zbl 1252.18028

[Krause and Štovíček 2010] H. Krause and J. Štovíček, "The telescope conjecture for hereditary rings via ext-orthogonal pairs", Adv. Math. 225:5 (2010), 2341-2364. MR 2011j:16013 Zbl 1242.16007

[Neeman 1992] A. Neeman, "The chromatic tower for $D(R)$ ", Topology 31:3 (1992), 519-532. MR 93h:18018 Zbl 0793.18008

[Neeman 1996] A. Neeman, "The Grothendieck duality theorem via Bousfield's techniques and Brown representability”, J. Amer. Math. Soc. 9:1 (1996), 205-236. MR 96c:18006 Zbl 0864.14008

[Neeman 2001] A. Neeman, Triangulated categories, Annals of Mathematics Studies 148, Princeton University Press, 2001. MR 2001k:18010 Zbl 0974.18008

[Ravenel 1984] D. C. Ravenel, "Localization with respect to certain periodic homology theories", Amer. J. Math. 106:2 (1984), 351-414. MR 85k:55009 Zbl 0586.55003

[Stevenson 2013] G. Stevenson, "Support theory via actions of tensor triangulated categories", $J$. Reine Angew. Math. 681 (2013), 219-254. MR 3181496 Zbl 1280.18010

[Stevenson 2014] G. Stevenson, "Subcategories of singularity categories via tensor actions", Compos. Math. 150:2 (2014), 229-272. MR 3177268 Zbl 1322.18004

[Thomason 1997] R. W. Thomason, "The classification of triangulated subcategories", Compos. Math. 105:1 (1997), 1-27. MR 98b:18017 Zbl 0873.18003

Received July 3, 2015. Revised October 28, 2015.

BENJAMIN ANTIEAU

Department of Mathematics, Statistics, And Computer Science

UNIVERSITY OF ILLINOIS AT CHICAGO

$851 \mathrm{~S}$. Morgan ST.

CHICAGO, IL 60607

UNITED STATES

benjamin.antieau@gmail.com

\section{GREG STEVENSON}

FACULTY OF MATHEMATICS

UNIVERSITÄT BIELEFELD

UNIVERSITÄTSTRASSE 25

D-33615 BIELEFELD

GERMANY

gstevens@math.uni-bielefeld.de 


\title{
PACIFIC JOURNAL OF MATHEMATICS
}

Founded in 1951 by E. F. Beckenbach (1906-1982) and F. Wolf (1904-1989)

$$
\text { msp.org/pjm }
$$

\section{EDITORS}

\author{
Don Blasius (Managing Editor) \\ Department of Mathematics \\ University of California \\ Los Angeles, CA 90095-1555 \\ blasius@math.ucla.edu
}

\author{
Paul Balmer \\ Department of Mathematics \\ University of California \\ Los Angeles, CA 90095-1555 \\ balmer@math.ucla.edu \\ Robert Finn \\ Department of Mathematics \\ Stanford University \\ Stanford, CA 94305-2125 \\ finn@math.stanford.edu \\ Sorin Popa \\ Department of Mathematics \\ University of California \\ Los Angeles, CA 90095-1555 \\ popa@math.ucla.edu
}

\author{
Vyjayanthi Chari \\ Department of Mathematics \\ University of California \\ Riverside, CA 92521-0135 \\ chari@math.ucr.edu \\ Kefeng Liu \\ Department of Mathematics \\ University of California \\ Los Angeles, CA 90095-1555 \\ liu@math.ucla.edu \\ Igor Pak \\ Department of Mathematics \\ University of California \\ Los Angeles, CA 90095-1555 \\ pak.pjm@gmail.com \\ Paul Yang \\ Department of Mathematics \\ Princeton University \\ Princeton NJ 08544-1000 \\ yang@math.princeton.edu
}

\section{PRODUCTION}

Silvio Levy, Scientific Editor, production@msp.org

\section{SUPPORTING INSTITUTIONS}

ACADEMIA SINICA, TAIPEI

CALIFORNIA INST. OF TECHNOLOGY

STANFORD UNIVERSITY

UNIV. OF BRITISH COLUMBIA

UNIV. OF CALIFORNIA, BERKELEY

UNIV. OF CALIFORNIA, DAVIS

UNIV. OF CALIFORNIA, LOS ANGELES

UNIV. OF CALIFORNIA, RIVERSIDE

UNIV. OF CALIFORNIA, SAN DIEGO

UNIV. OF CALIF., SANTA BARBARA
KEIO UNIVERSITY

MATH. SCIENCES RESEARCH INSTITUTE

NEW MEXICO STATE UNIV.

OREGON STATE UNIV.
Daryl Cooper

Department of Mathematics

University of California

Santa Barbara, CA 93106-3080 cooper@math.ucsb.edu

Jiang-Hua Lu

Department of Mathematics

The University of Hong Kong

Pokfulam Rd., Hong Kong

jhlu@maths.hku.hk

$$
\text { Jie Qing }
$$

Department of Mathematics

University of California

Santa Cruz, CA 95064

qing@cats.ucsc.edu

\author{
UNIV. OF CALIF., SANTA CRUZ \\ UNIV. OF MONTANA \\ UNIV. OF OREGON \\ UNIV. OF SOUTHERN CALIFORNIA \\ UNIV. OF UTAH \\ UNIV. OF WASHINGTON \\ WASHINGTON STATE UNIVERSITY
}

These supporting institutions contribute to the cost of publication of this Journal, but they are not owners or publishers and have no responsibility for its contents or policies.

See inside back cover or msp.org/pjm for submission instructions.

The subscription price for 2016 is US \$/year for the electronic version, and \$/year for print and electronic.

Subscriptions, requests for back issues and changes of subscriber address should be sent to Pacific Journal of Mathematics, P.O. Box 4163, Berkeley, CA 94704-0163, U.S.A. The Pacific Journal of Mathematics is indexed by Mathematical Reviews, Zentralblatt MATH, PASCAL CNRS Index, Referativnyi Zhurnal, Current Mathematical Publications and Web of Knowledge (Science Citation Index).

The Pacific Journal of Mathematics (ISSN 0030-8730) at the University of California, c/o Department of Mathematics, 798 Evans Hall \#3840, Berkeley, CA 94720-3840, is published twelve times a year. Periodical rate postage paid at Berkeley, CA 94704, and additional mailing offices. POSTMASTER: send address changes to Pacific Journal of Mathematics, P.O. Box 4163, Berkeley, CA 94704-0163.

PJM peer review and production are managed by EditFLOW ${ }^{\circledR}$ from Mathematical Sciences Publishers.

PUBLISHED BY

\section{I. mathematical sciences publishers}

nonprofit scientific publishing

http://msp.org/

(C) 2016 Mathematical Sciences Publishers 


\section{PACIFIC JOURNAL OF MATHEMATICS}

Volume $283 \quad$ No. $1 \quad$ July 2016

A New family of simple $\mathfrak{g l}_{2 n}(\mathbb{C})$-modules

JONATHAN NILSSON

Derived categories of representations of small categories over commutative noetherian rings

BENJAMIN ANTIEAU and GREg STEVENSON

Vector bundles over a real elliptic curve

INDRANIL BISWAS and FLORENT SCHAFFHAUSER

$\mathrm{Q}(\mathrm{N})$-graded Lie superalgebras arising from fermionic-bosonic representations

JIN CHENG

Conjugacy and element-conjugacy of homomorphisms of compact Lie groups

YiNGJUE FANG, GANG HAN and BINYONG SUN

Entire sign-changing solutions with finite energy to the fractional Yamabe equation

DANILO GARRIDO and MoniCa Musso

Calculation of local formal Mellin transforms

ADAM GRAHAM-SQUIRE

The untwisting number of a knot

KENAN INCE

A Plancherel formula for $L^{2}(G / H)$ for almost symmetric subgroups

Bent Ørsted and Birgit SPEH

Multiplicative reduction and the cyclotomic main conjecture for $\mathrm{GL}_{2}$

CHRISTOPHER SKINNER

Commensurators of solvable $S$-arithmetic groups

\section{DANIEL STUDENMUND}

Gerstenhaber brackets on Hochschild cohomology of quantum symmetric algebras and their group extensions

SARAH WITHERSPOON and GUODONG ZHOU 